\title{
Problemas de M-partição em cografos
}

\author{
Raquel de Souza Francisco Bravo ${ }^{1}$, Maria Luíza López da $\mathrm{Cruz}^{1}$ \\ ${ }^{1}$ Departamento de Computação - Universidade Federal Fluminense (UFF) \\ Niterói - RJ - Brasil \\ raquel@ic.uff.br, marialopezeid.uff.br
}

\begin{abstract}
The study of graphs is an important topic in Computer Science, in theoretical and practical terms. This project consists in solving a problem in the area of graph theory: the M-partition problem seeks a characterization of the graphs whose set of vertices can be partitioned into $k$ independent sets and $l$ cliques, finding the minimal obstructions for the partition. Due to the difficulty of the M-partition problem, which is NP-complete, this work considers the problem restricted to the class of cographs with a symmetric matrix $M$ of order $4(m=4)$. This matrix represents the external and internal restrictions on the desired partition and its entries can be elements $\{0,1, *\}$.
\end{abstract}

Resumo. O estudo de grafos é um tópico importante em Ciência da Computação, no campo teórico e prático. Este trabalho consiste na resolução de um problema na área de teoria dos grafos: o problema da M-partição visa apresentar uma caracterização dos grafos cujo conjunto de vértices pode ser particionado em $k$ conjuntos independentes e l cliques, encontrando as obstruções minimais para essa partição. Devido à dificuldade do problema da M-partição, que é NP-completo, este trabalho considera o problema restrito à classe dos cografos com matriz simétrica $M$ de ordem $4(m=4)$. Esta matriz representa as restrições externas e internas acerca da partição desejada e suas entradas podem ser elementos $\{0,1, *\}$.

\section{Introdução}

Os grafos estão presentes em nossas vidas e nos auxiliam em inúmeras situações do cotidiano, como no acesso à internet, na navegação entre sites, minimização de despesas, ou seja, decisões que requerem otimização e que em sua maioria podem ser resolvidas, de maneira eficiente, pelo uso de algoritmos.

\subsection{Problemas de Partição em Grafos}

Problemas de partição em grafos têm despertado interesse devido às pesquisas em grafos perfeitos [Golumbic 1980] (grafos cujo número cromático é igual ao tamanho da maior clique, para todo subgrafo induzido) e também pela procura de algoritmos eficientes de reconhecimento de determinadas classes de grafos. Muitos desses problemas de partição em grafos podem ser descritos como tendo por objetivo particionar o conjunto de vértices de um grafo em conjuntos $V_{1}, V_{2}, \ldots, V_{m}$, onde $V_{1} \cup V_{2} \cup \ldots \cup V_{m}=V$ e $V_{i} \cap V_{j}=\emptyset$, para todo $i \neq j, i, j \leq m$, exigindo-se, porém, algumas propriedades sobre estes conjuntos de vértices, que podem ser internas, como, por exemplo, exigir que os vértices de cada conjunto $V_{i}$ sejam dois a dois adjacentes ( $V_{i}$ é uma clique) ou dois a dois não-adjacentes ( $V_{i}$ é um conjunto independente), ou externas, onde as exigências são feitas sobre os pares $\left(V_{i}, V_{j}\right)$, isto é, $V_{i}$ e $V_{j}$ podem ser completamente adjacentes ou não-adjacentes entre si. Um conjunto de vértices $I$ de um grafo $G$ é um conjunto independente se $G[I]$ é um grafo sem arestas. Um conjunto independente $I$ é dito maximal se para qualquer conjunto independente $I^{\prime}$ tal que $I$ contém $I^{\prime}$ então $I=I^{\prime}$. Denotamos por $I_{n}$ um conjunto independente de $n$ vértices. Por outro lado, um conjunto de vértices $B$ de um grafo $G$ é uma clique se $G[B]$ é um grafo completo. Denotamos por $K_{n}$ uma clique de $n$ vértices. Como 
exemplo, citamos o problema da $k$-coloração, onde desejamos particionar o conjunto de vértices de um grafo em $k$ conjuntos independentes $V_{1}, \ldots, V_{k}$ (sem restrições externas). Sabe-se que esse problema pode ser resolvido em tempo polinomial para $m \leq 2$ e é NP-completo para $m \geq 3$.

\subsubsection{O Estado da Arte}

Em [Brandstädt 1996] Brandstädt definiu uma nova classe de grafos inserida no contexto de partição de grafos, a classe dos grafos $(k, \ell)$ : grafos cujo conjunto de vértices pode ser particionado em $k$ conjuntos independentes e $\ell$ cliques, havendo a possibilidade de alguns desses conjuntos serem vazios. Denotamos por grafos $(k, 0)^{*}$, grafos cujo conjunto de vértices pode ser particionado em $k$ conjuntos independentes e nenhuma clique, não havendo restrição externa entre os conjuntos independentes. Para o problema desta classe de grafos, grafos $(k, \ell)$, o problema da M-partição equivalente tem a matriz simétrica $M$ de ordem $k+\ell$ com $k$ elementos da diagonal principal nulos, $\ell$ elementos da diagonal principal iguais a 1 e elementos do tipo $*$ só aparecem fora da diagonal principal. Brandstädt considerou em particular as classes dos grafos $(2,1)$, grafos $(1,2)$ e grafos $(2,2)$, apresentando algoritmos polinomiais para reconhecer estas classes [Brandstädt 1996, Brandstädt 1998]. Feder, Hell, Klein e Motwani [Feder et al. 1999] também apresentaram algoritmos polinomiais para o reconhecimento destas classes que surgiram como sub-produto de algoritmos de partição em subgrafos densos e esparsos. Por outro lado, sabese que o reconhecimento de grafos $(k, \ell)$ para $k \geq 3$ ou $\ell \geq 3$ é um problema NP-completo [Brandstädt 1996]. Em vista deste resultado de NP-completude, restringimos este problema à classe dos cografos. Um grafo é um cografo se não possui caminho induzido de comprimento 3. Em [Bravo 2006, Bravo et al. 2005, Bravo et al. 2011] é apresentada uma caracterização por subgrafos proibidos para a classe dos cografos $(k, \ell)$ com restrições externas entre as partes do tipo *, bem como um algoritmo linear para o reconhecimento desta classe de grafos.

Neste trabalho, consideramos o problema da $M$-partição em grafos, que foi introduzido por Feder et al. [Feder et al. 1999]. Para cada matriz simétrica $M$ definida sobre $\{0,1, *\}$, o problema da $M$-partição tem como objetivo encontrar uma partição de um grafo de entrada em conjuntos independentes, cliques ou conjuntos arbitrários, impondo-se algumas restrições entre esses conjuntos. A matriz simétrica $M$ possui elementos $M(i, i)$, que representam a restrição interna no conjunto $V_{i}$ (se $M(i, i)=0$, então, $V_{i}$ é um conjunto independente; se $M(i, i)=1$, então, $V_{i}$ é uma clique) e cada elemento fora da diagonal principal $M(i, j), i \neq j$, representa uma restrição externa entre os conjuntos $V_{i}$ e $V_{j}$ (se $M(i, j)=0$, então, $V_{i}$ e $V_{j}$ são completamente não adjacentes; se $M(i, j)=1$, então, $V_{i}$ e $V_{j}$ são completamente adjacentes; se $M(i, j)=*$, então, não há nenhuma restrição sobre as arestas entre $V_{i}$ e $V_{j}$ ).

Os grafos que admitem uma partição dos vértices de forma a respeitar as restrições impostas entre as partes (representadas na matriz) são denominados $M$-particionáveis. Quando um grafo $G$ não é $M$-particionável, dizemos que este é uma $M$-obstrução. Em particular, uma $M$-obstrução $H$ é minimal quando $H-v$ é $M$-particionável para qualquer vértice $v$ de $H$.

$\mathrm{O}$ intuito deste trabalho é determinar as $M$-obstruções minimais de classes de grafos com poucos $P_{4}$ 's induzidos. Ele apresenta uma continuação de dois trabalhos: [Viana 2013], no qual se determina as $M$-obstruções minimais quando $M$ é uma matriz de ordem no máximo três para a classe dos cografos e, [Leite 2013], que determina as $M$-obstruções minimais dos cografos quando $M$ é uma matriz de ordem quatro com diagonal nula (particionar em quatro conjuntos independentes).

\section{Resultados Encontrados}

Antes de apresentarmos a prova de um dos teoremas contidos neste trabalho, vale descrevermos que o intuito é encontrarmos todas as $M$-obstruções minimais dos cografos quando $M$ é uma matriz de ordem quatro com diagonal principal com três 0's e um 1, ou seja, analisar as 
partições do conjunto de vértices em 3 conjuntos independentes e 1 clique, com todas as possíveis restrições externas. Para iniciar, obtemos os resultados para todas as restrições externas possíveis envolvendo a clique e nenhuma restrição externa entre os conjuntos independentes. Provaremos, agora, um dos casos analisados durante o desenvolvimento deste trabalho, o caso $(3,1)^{5}$. Este caso é referente a M-partição onde temos as restrições externas entre a clique e os três conjuntos independentes do tipo 0 , nenhum vértice da clique possui aresta a qualquer vértice pertencente a um dos conjuntos independentes, e as restrições externas entre os conjuntos independentes do tipo *, conforme figura 1 abaixo.

Teorema 1 [Viana 2013] Seja G um cografo, G é um grafo $(2,0)^{*}\left((3,0)^{*, *, *}\right)$ se e somente se $G$ não contém $K_{3}\left(K_{4}\right)$ como subgrafo induzido.

Teorema 2 Seja G um cografo, G é um grafo $(3,1)^{5}$ se e somente se $G$ não contém nenhum dos grafos da Figura 2 como subgrafo induzido.

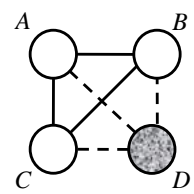

Figura 1. $\boldsymbol{M}$-partição- $(3,1)^{5}$

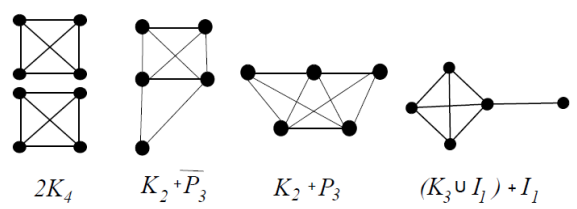

Figura 2. Grafos Proibidos para a $\boldsymbol{M}$-partição- $(3,1)^{5}$

Prova. $(\Rightarrow)$ Podemos verificar facilmente que se $G$ contém qualquer um dos grafos da Figura 2 como subgrafo induzido, por contraposição, então $G$ não é um cografo $(3,1)^{5}$. Pode-se também verificar que cada um desses grafos é minimal, já que a retirada de qualquer um de seus vértices torna-o $(3,1)^{5}$.

$(\Leftarrow)$ Seja $G$ um cografo que é minimalmente não $(3,1)^{5}$, ou seja, para todo $v \in V(G)$, $G-v$ é um grafo $(3,1)^{5}$. Suponha, por contradição, que $G$ não contém nenhum dos grafos da Figura 2 como subgrafo induzido. Como $G$ é cografo, temos que $G$ ou $\bar{G}$ é desconexo, [Jamison and Olariu 1995]. No que segue, analisaremos os dois casos:

(i) $G$ é desconexo. Assim, $G=G_{1} \cup G_{2} \cup \ldots \cup G_{k}$, com $k \geq 2$, onde cada $G_{i}$ é trivial ou conexo. Caso $G_{i}$ seja $(3,0)^{*, *, *}$, para algum $i=1,2, \ldots, k$, então $G$ é $(3,1)^{5}$, pois $G-G_{i}$ é $(3,1)^{5}$ por minimalidade. De fato, $G-G_{i}$ pode ser particionado em $\left(S_{1}, S_{2}, S_{3}, K\right)$, onde $S_{1}, S_{2}, S_{3}$ são conjuntos independentes e $K$ é a clique da partição. Da mesma forma, temos $G_{i}$ com partição $\left(S_{1}^{*}, S_{2}^{*}, S_{3}^{*}\right)$, já que $G_{i}$ é $(3,0)^{*, *, *}$. Assim, pode-se perceber que o grafo $G$ será $(3,1)^{5}$ com partição $\left(S_{1} \cup S_{1}^{*}, S_{2} \cup S_{2}^{*}, S_{3} \cup S_{3}^{*}, K\right)$. Contradição! Logo, pelo Teorema 1 cada $G_{i}$ contém um $K_{4}$ e portanto $G$ contém um $2 K_{4}$ induzido. Absurdo!

(ii) $\bar{G}$ é desconexo. Pela propriedade da decomposição modular, [Jamison and Olariu 1995], temos que $G=G_{1}+G_{2}+\ldots+G_{k}$, com $k \geq 2$ e cada $G_{i}$ sendo trivial ou desconexo. Desta forma, analisaremos três subcasos ( $k \geq 4, k=3$ e $k=2)$. Para $k \geq 4$ : Se $G_{i}$ for trivial, para todo $i=1, \ldots, k$, então $G$ é $(3,1)^{5}$, já que $G_{1}+G_{2}+\ldots+G_{k}$ forma uma clique. Contradição. Suponha que apenas um dos $G_{i}$ 's para $i=1, \ldots, k$ contém $I_{2}$. Desse modo, $G$ contém $I_{2}+I_{1}+I_{1}+I_{1} \cong K_{2}+P_{3}$. Contradição. Caso algum $G_{i}$ contenha 
aresta, podemos observar que $G_{i}$ contém $\overline{P_{3}}$, já que $G_{i}$ é desconexo e, portanto, contém $I_{2}$. Neste caso, a análise é semelhante ao caso anterior. Resta analisar o caso em que dois dos $G_{i}$ 's contém $I_{2}$. Assim, temos que $G$ contém $I_{2}+I_{2}+I_{1}+I_{1} \cong K_{2}+P_{3}$. Contradição. Para $k=3$ : Temos $G=G_{1}+G_{2}+G_{3}$. Se, para todo $i=1,2,3, G_{i}$ for tal que $V\left(G_{i}\right)$ seja um conjunto independente temos que $G$ é $(3,0)^{*, *, *}$ e portanto $(3,1)^{5}$, pois desse modo consegue-se colocar $G_{1}, G_{2}$ e $G_{3}$ em cada um dos conjuntos independentes da partição. Absurdo! Resta analisar o caso em que algum $G_{i}$ contém aresta. Neste caso, $G$ possuirá $I_{1}+\bar{P}_{3}+I_{1} \cong K_{2}+\bar{P}_{3}$. Absurdo! Para $k=2$ : Temos $G=G_{1}+G_{2}$. Se $G_{1}$ e $G_{2}$ são tais que $V\left(G_{1}\right)$ e $V\left(G_{2}\right)$ são conjuntos independentes, então $G$ é $(3,1)^{5}$, pois desse modo é possível colocar $G_{1}$ e $G_{2}$ em cada um dos conjuntos independentes da partição. Absurdo! Se apenas um $G_{i}$ é tal que $V\left(G_{i}\right)$ é um conjunto independente, suponhamos, sem perda de generalidade, que seja $G_{1}$. Temos que $G_{2}$ não pode ser $(2,0)^{*}$, caso contrário $G$ é um grafo $(3,1)^{5}$. Contradição. Logo, pelo Teorema 1 , $G_{2}$ contém um $K_{3}$. Assim, $G$ contém $\left(K_{3} \cup I_{1}\right)+I_{1}$. Absurdo. Por fim, analisamos o caso em que ambos $G_{1}$ e $G_{2}$ possuem arestas. Nesse caso, como $G_{1}$ e $G_{2}$ são desconexos, temos que $G_{1}$ e $G_{2}$ possuem $\bar{P}_{3}$. Assim, $G$ contém $K_{2}+\bar{P}_{3}$. Absurdo!

Concluímos que o cografo $G$ é $(3,1)^{5}$ se e somente se não contém nenhum dos grafos $2 K_{4}, K_{2}+P_{3}, K_{2}+\bar{P}_{3}$ e $\left(K_{3} \cup I_{1}\right)+I_{1}$ como subgrafo induzido.

\section{Conclusão}

O trabalho determina as $M$-obstruções minimais para cografos quando $M$ é uma matriz de ordem quatro com diagonal constituída por três elementos 0 e um único elemento 1 , contendo restrições externas apenas entre os conjuntos independentes e a clique. Por motivo de falta de espaço, apresentamos a prova de apenas um dos casos.

\section{Referências}

Brandstädt, A. (1996). Partitions of graphs into one or two independent sets and cliques. Discrete Mathematics, 152(1-3):47-54.

Brandstädt, A. (1998). Corrigendum. Discrete Mathematics, 186:295.

Bravo, R. S. F. (2006). Particionamento de cografos em conjuntos independentes e cliques. Dissertação de mestrado, COPPE/UFRJ, Rio de Janeiro, RJ, Brasil.

Bravo, R. S. F., Klein, S., and Nogueira, L. (2005). Characterizing $(k, \ell)$-partitionable cographs. Eletronic Notes on Discrete Mathematics, 22:277-280.

Bravo, R. S. F., Klein, S., Nogueira, L., and Protti, F. (2011). Characterization and recognition of $\mathrm{P}_{4}$-sparse graphs partitionable into independent sets and cliques. Discrete Applied Mathematics, 159:165-173.

Feder, T., Hell, P., Klein, S., and Motwani, R. (1999). Complexity of graph partition problems. In F. W. Thatcher, R. E. M., editor, Proceedings of the 31st Annual ACM Symposium on the Theory of Computing (STOC'99), pages 464-472. Plenum Press.

Golumbic, M. C. (1980). Algorithmic graph theory and perfect graphs. Academic Press, New York.

Jamison, B. and Olariu, S. (1995). p-components and the homogeneous decomposition of graphs. SIAM Journal on Discrete Mathematics, 8:448-463.

Leite, J. S. S. (2013). Caracterização dos cografos- $(4,0)$ por subgrafos proibidos com restrições externas. Dissertação de mestrado, UFF, Niterói, RJ, Brasil.

Viana, C. C. (2013). M M $3 \times 3$-obstrução minimal de cografos. Dissertação de mestrado, UFF, Niterói, RJ, Brasil. 